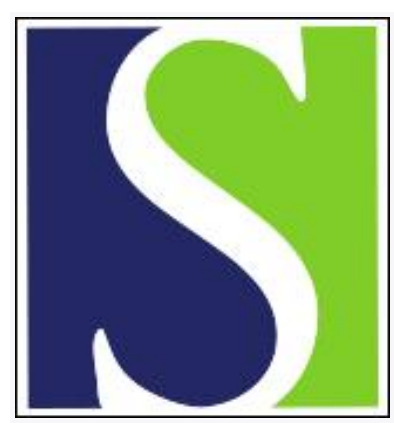

Scand J Work Environ Health 1983;9(2):148-154

https://doi.org/10.5271/sjweh.2431

Issue date: Apr 1983

Uses of computer-generated maps in occupational hazard and mortality surveillance.

by Frazier TM, Lalich NR, Pedersen DH

This article in PubMed: www.ncbi.nlm.nih.gov/pubmed/6648412

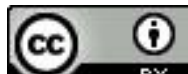




\title{
Uses of computer-generated maps in occupational hazard and mortality surveillance
}

\author{
by Todd M Frazier, ScM, Nina R Lalich, MSPH, David H Pedersen, MS ${ }^{1}$
}

\begin{abstract}
FRAZIER TM, LALICH NR, PEDERSEN DH. Uses of computer-generated maps in occupational hazard and mortality surveillance. Scand $j$ work environ health 9 (1983) 148-154. The purpose of the present paper was to describe five ways in which computergenerated maps for 3,073 United States political subdivisions (counties) are being used as a part of the National Institute for Occupational Safety and Health surveillance system. Four maps show the approximate spatial location of potential workplace hazards in terms of (i) worksites, (ii) workers, (iii) estimates of the proportion of workers at risk for US counties, and (iv) counties within a selected state.. A fifth map shows age-standardized county-level mortality for a cause of death associated with an occupational risk. These five types of maps represent first efforts to present and disseminate information in an effective format and to generate occupational health surveillance leads that may warrant further research.
\end{abstract}

Key terms: chemicals in the workplace, computer mapping.

In the United States, the National Institute for Occupational Safety and Health (NIOSH) has the responsibility for developing a surveillance system that will contribute to the detection and description of epidemiologically significant changes in work-related injury, disease, disability, and mortality. The scope of this surveillance system is broad. It involves the assessment of hazards and illnesses among 106 million American workers employed in nearly 5 million worksites scattered over a land area of 3.5 million square miles (9.1 million $\mathrm{km}^{2}$ ).

Occupational health surveillance at $\mathrm{NIOSH}$ involves both the identification of workplace hazards and the effects of hazards on the health of workers. Surveillance is one of the methods used for risk identification in the priority setting process described by Rantanen (7).

1 Division of Surveillance Hazard Evaluations and Field Studies, National Institute for Occupational Safety and Health, Cincinnati, Ohio, United States.

Reprint requests to: Mr TM Frazier, National Institute for Occupational Safety and Health, Robert A Taft Laboratories, 4676 Columbia Parkway, Cincinnati, OH 45226, USA.
Funds for this type of surveillance are limited, amounting to approximately two cents (USD 0.02) per worker per year. Therefore it is necessary to develop efficient information collection, analysis, and dissemination methods. The purpose of this paper is to describe one of these methods, the use of computer-generated maps in the surveillance of work-related hazards and health effects. In order to provide a background for the description, we will first review the origin of NIOSH interests in the use of computerized mapping in surveillance.

Interest in the application of computergenerated maps to descriptive epidemiology in the United States began in the early 1960 s with the work of Stark at the National Cancer Institute (4). Mason and his colleagues, also working at the National Cancer Institute, moved ahead rapidly during the 1970 s with their publication of maps depicting patterns of cancer mortality (3). Our mapping work at NIOSH began as a result of the funding and technical assistance we received from the National Cancer Institute. With the National Cancer Institute, the National Center for Health Statistics, and other 
research institutes we share an interest in developing methods that allow us to study county-level mortality and occupational hazard profiles.

In the United States the National Center for Health Statistics compiles vital statistics from all states. Aggregate data tapes are used to describe county-level cause-specific and age-standardized mortality rates. This information has been used by several investigators to map cancer, cardiovascular, and infant mortality rates $(1,2)$. At NIOSH we have also used mortality data to map causes of death known to be associated with occupation. During this process we recognized however the need and opportunity for producing maps that generate surveillance leads by identifying counties in which the workforce has a high potential for exposure to occupational hazards. An inventory of chemical, physical, and biological hazards has therefore been developed from the National Occupational Hazard Survey that was conducted by NIOSH during 19721974 (5).

In this survey a national probability sample of workplaces was selected for the purpose of gathering information on potential exposure of workers to hazards. Over the years $1972-1974,20$ surveyors visited over 4,500 different industrial sites throughout the United States. Each site visit consisted of a management interview to gather basic information about the facility and its occupational health policies and a detailed walk-through survey of the plant. During the walk-through survey all observed exposures to specific chemicals, tradename products, and physical agents were recorded as "potential exposures." The surveyors also noted the duration of potential exposures and any industrial hygiene control measures that were being applied. The final result of the survey effort was the National Occupational Hazard Survey data base, consisting of almost 5 million records describing potential workplace exposures by industry, occupation, and agent. This data file provided the first step in generating county-level hazard maps.

The second step involved the use of a commercially available computerized file that describes the characteristics of about 4.7 million American industrial facilities. This is the Dun \& Bradstreet file. The Dun \& Bradstreet descriptors include standard industrial codes, county in which the workplace is located, and size of workforce. The National Occupational Hazard Survey and the Dun \& Bradstreet file have been linked. We have assumed that the types of potential exposures seen in the Survey for a specified standard industrial code are representative of all similar standard industrial codes in the file. By using this linked data base, we are able to generate county-level maps that approximate the geographic location of worksites with a high probability of workers potentially exposed to a selected industrial hazard. We are well aware of the approximate nature of these maps. In some respects they may be about as predictive as a long-range map of weather conditions. Nevertheless we believe that they will be useful in generating hypotheses and in stimulating questions and leads for further investigation.

With this information as background we will next describe five types of maps that we have recently developed at NIOSH. The first four maps describe potential exposures to a workplace hazard. The fifth type of map displays county-level cause-specific mortality rates for a disease that is in part caused by this hazard.

\section{Type 1 - Worksites/specific hazard}

Since 1975 NIOSH has published a series of 37 current intelligence bulletins that review, evaluate, and disseminate new information that may indicate either the existence of an occupational hazard not previously recognized or provide new information about a known hazard. These bulletins convey important public health information and recommend voluntary protective action.

In collaboration with the NIOSH staff responsible for preparing the bulletins we are exploring the utility of adding a county-level computer-generated map to each publication. This map will show the location of worksites similar in classification to those where the agent specified in the bulletin was observed during the 


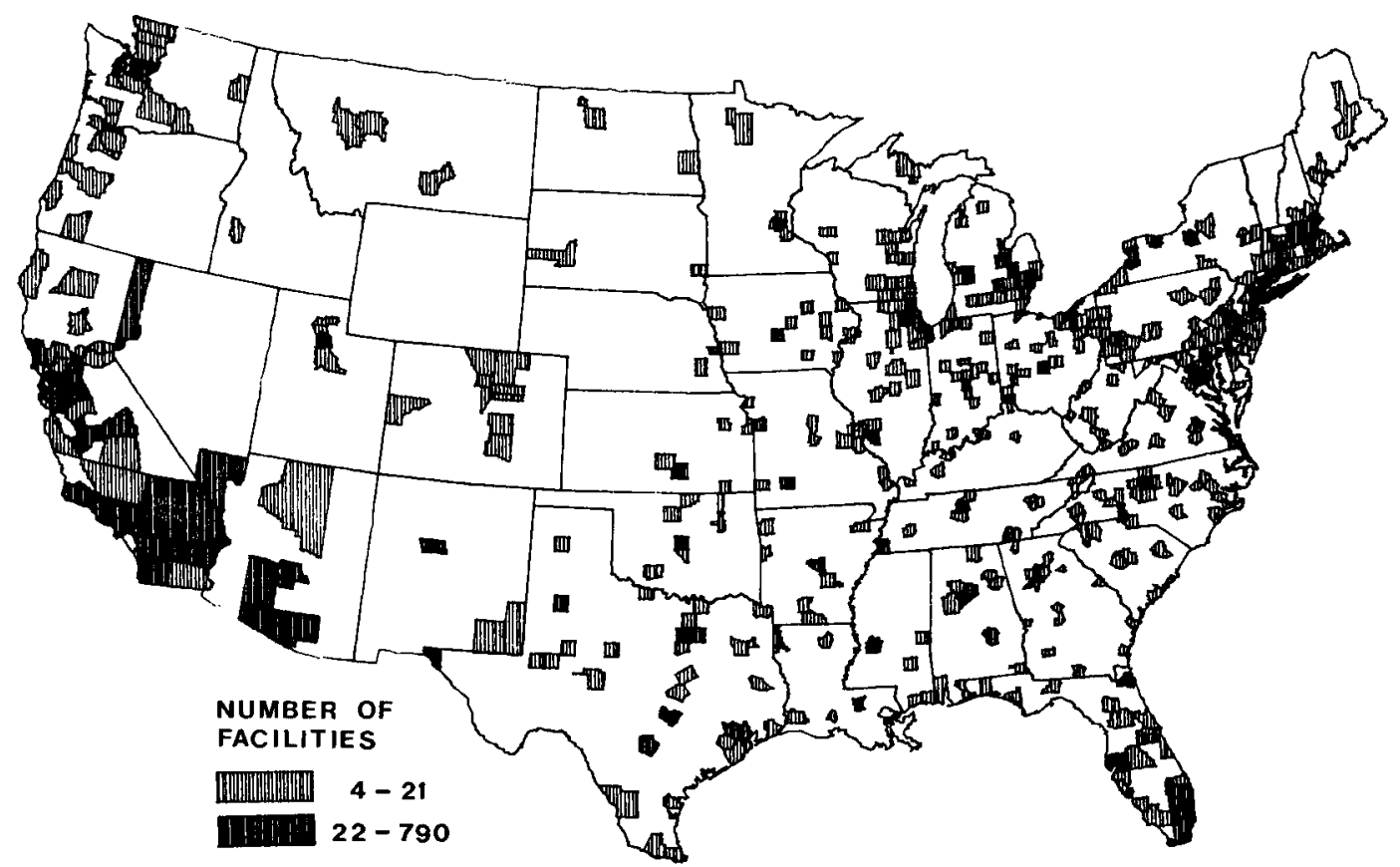

Fig 1. Distribution of facilities where workers are potentially exposed to formaldehyde. Selection criterion: industries in which potential exposure was observed for $10 \%$ or more of the workforce (National Occupational Hazard Survey).

1972-1974 National Occupational Hazard Survey. For illustrative purposes the map in fig 1 shows county worksite distribution for a recent bulletin (6). It shows a computer-generated map of the 3,073 US counties coded to display the number of facilities (worksites) of the type in which formaldehyde was observed in the National Occupational Hazard Survey. There are two features of this map that need explanation. The first is the selection criterion that is stated as "Industries in which potential exposure was observed for $10 \%$ or more of the workforce." The density configuration of the map can be changed by varying this criterion. For example, if we portrayed worksites with $90 \%$ of the plant's workforce potentially exposed, very few counties, if any, would qualify. In contrast, if the criterion was set at $1 \%$, many more worksites would qualify, and the map would lose much of its discriminatory utility. On the basis of our experience with the chemical described in this bulletin we have selected a $10 \%$ exposure level.

Secondly, the selection of the class intervals used for coding counties can affect the visual impression conveyed by the map. In this map the intervals were selected according to cumulative percentile distribution points, ie, 60th percentile or less, 61st-89th percentile, and 90th percentile and over. Thus counties coded solid black in the formaldehyde map constitute the top $10 \%$ of all US counties with respect to the number of worksites in which $10 \%$ or more of the workers are potentially exposed to a chemical of high interest because of the evidence of its carcinogenicity.

Computer-generated county-level maps will complement the descriptions presently used in the current intelligence bulletins to show the production, use, and potential exposure to workplace hazards.

\section{Type 2 - Workers/specific hazards}

The next map (fig 2) shows the spatial distribution of workers rather than worksites. The example selected is again concerned with formaldehyde, but in this map there are two important changes. First the selection criterion has been set to show 


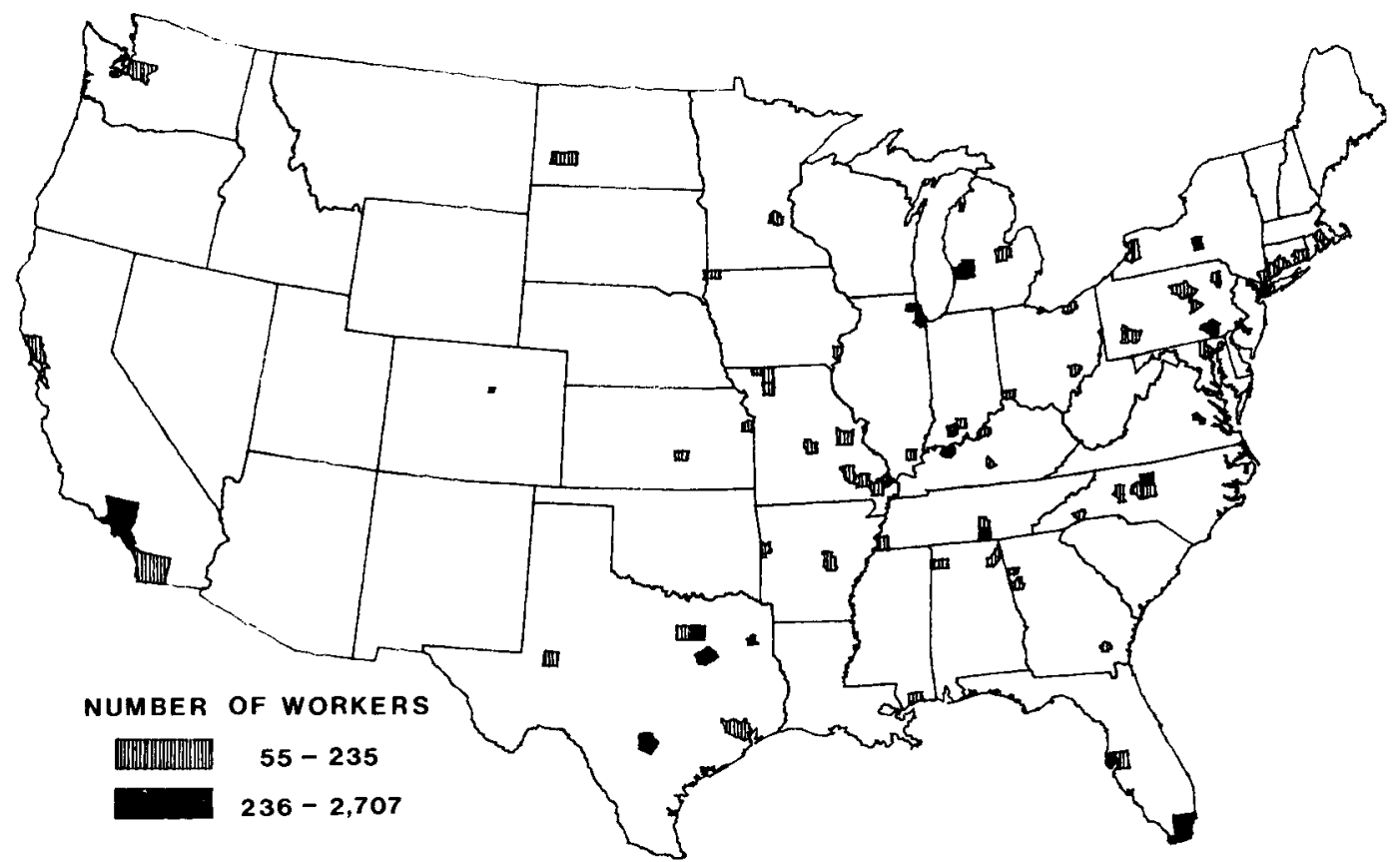

Fig 2. Distribution of workers potentially exposed to formaldehyde. Selection criterion: industries in which potential exposure was observed for $45 \%$ or more of the workforce (National Occupational Hazard Survey).

the county location of worksites in industries where $45 \%$ or more of the workforce is potentially exposed to formaldehyde. Second this map shows the number of workers potentially exposed to formaldehyde. Maps of this type may prove to be of use in identifying clusters of workers at risk of exposure to a specified industrial hazard.

The map in fig 2 was generated by automated cartographic techniques that use data from the two previously mentioned data files, the National Occupational Health Survey and the Dun \& Bradstreet file. The assumptions made in the use of both files make it necessary to reemphasize the approximate nature of these maps, particularly when we are making estimates of the number of workers potentially exposed to specific hazards.

\section{Type 3 - Proportion of workers/hazard}

If one is willing to assume that the Dun \& Bradstreet count of workers in a given county is reasonably complete, it is possible to generate county-level maps that estimate the proportion of workforce that is potentially exposed to a workplace hazard in a particular area. This type of estimate is useful when we are attempting to correlate occupational risks with county-based mortality patterns. Fig 3 shows, for counties, the number of workers potentially exposed to formaldehyde per 100,000 workers in that area. A map like this may suggest leads that warrant further epidemiologic investigation.

\section{Type 4 - Proportion of workers per hazard per state level}

Another series of maps can be generated. for individual states. In addition to providing greater detail, these maps are very useful in our efforts to decentralize occupational health surveillance and to help state health departments to improve their occupational health programs. Fig 4 shows the proportion of workers potentially exposed to formaldehyde in counties in the state of Indiana. 


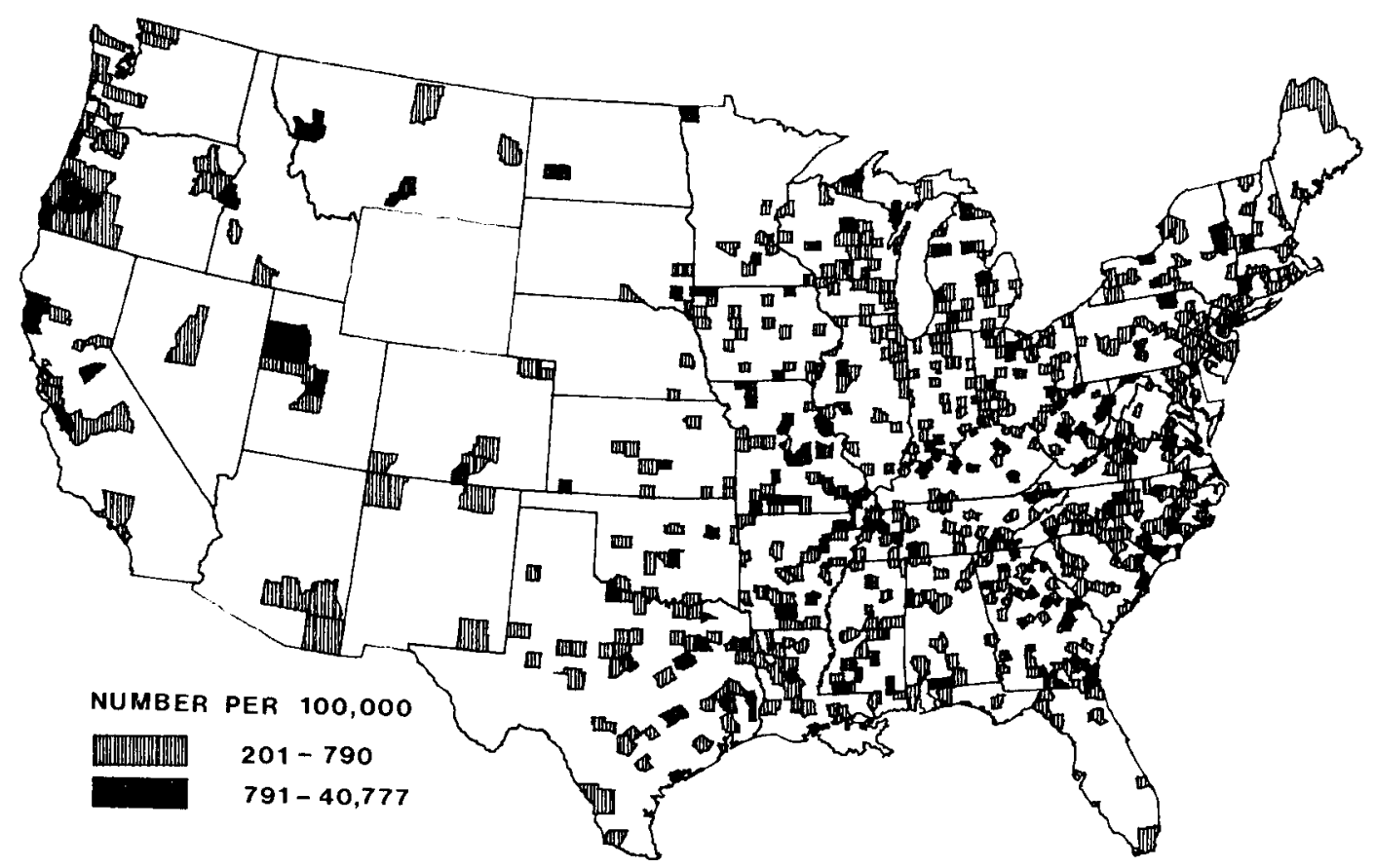

Fig 3. Distribution of workers potentially exposed to formaldehyde. Selection criterion: industries in which $5 \%$ or more of the workforce is potentially exposed (National Occupational Hazard Survey).

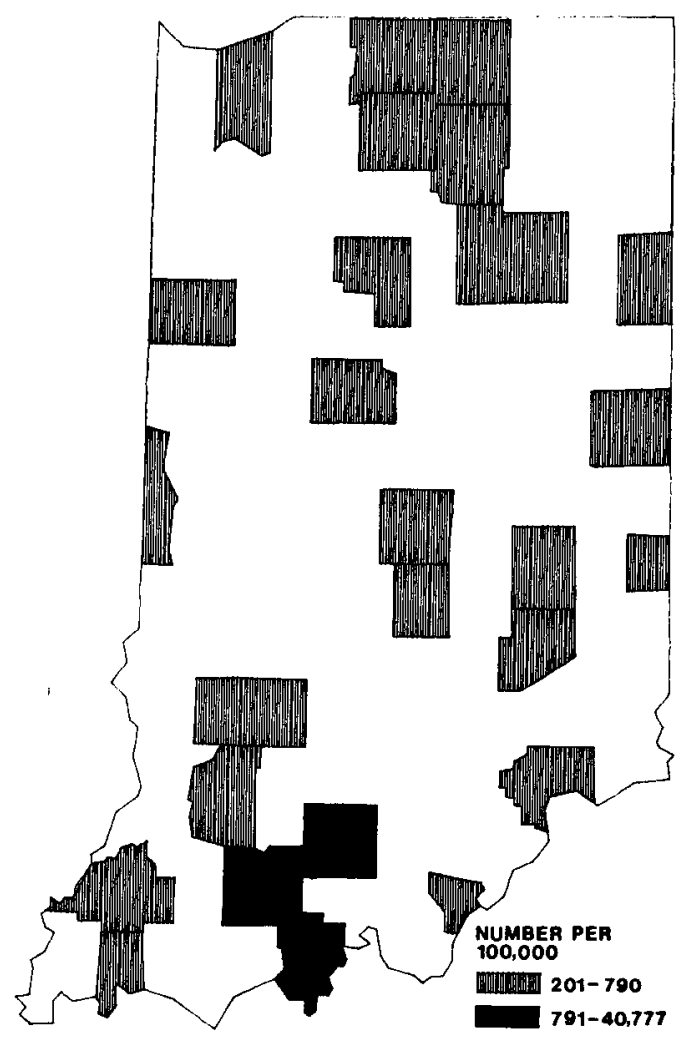

\section{Type 5 - Cause-specific county mortality rates}

As stated earlier, our surveillance efforts are concerned with both workplace hazards and work-related diseases, disabilities, and deaths. For the latter, we are attempting to use standardized countylevel cause-specific mortality rates as a means of displaying geographic patterns for causes of death that are related to occupational risks. For illustrative purposes the cause of death selected was that thought to be related to formaldehyde (fig 5). Other causes of death that are much more clearly related to occupational exposures could have been selected, eg, benzene and leukemia, but our purpose is not to demonstrate association but rather to display data in ways that suggest surveillance leads. Again, in

Fig 4. Distribution of workers potentially exposed to formaldehyde in the state of Indiana. Selection criterion: industries in which $5 \%$ or more of the workforce is potentially exposed (National Occupational Hazard Survey). 


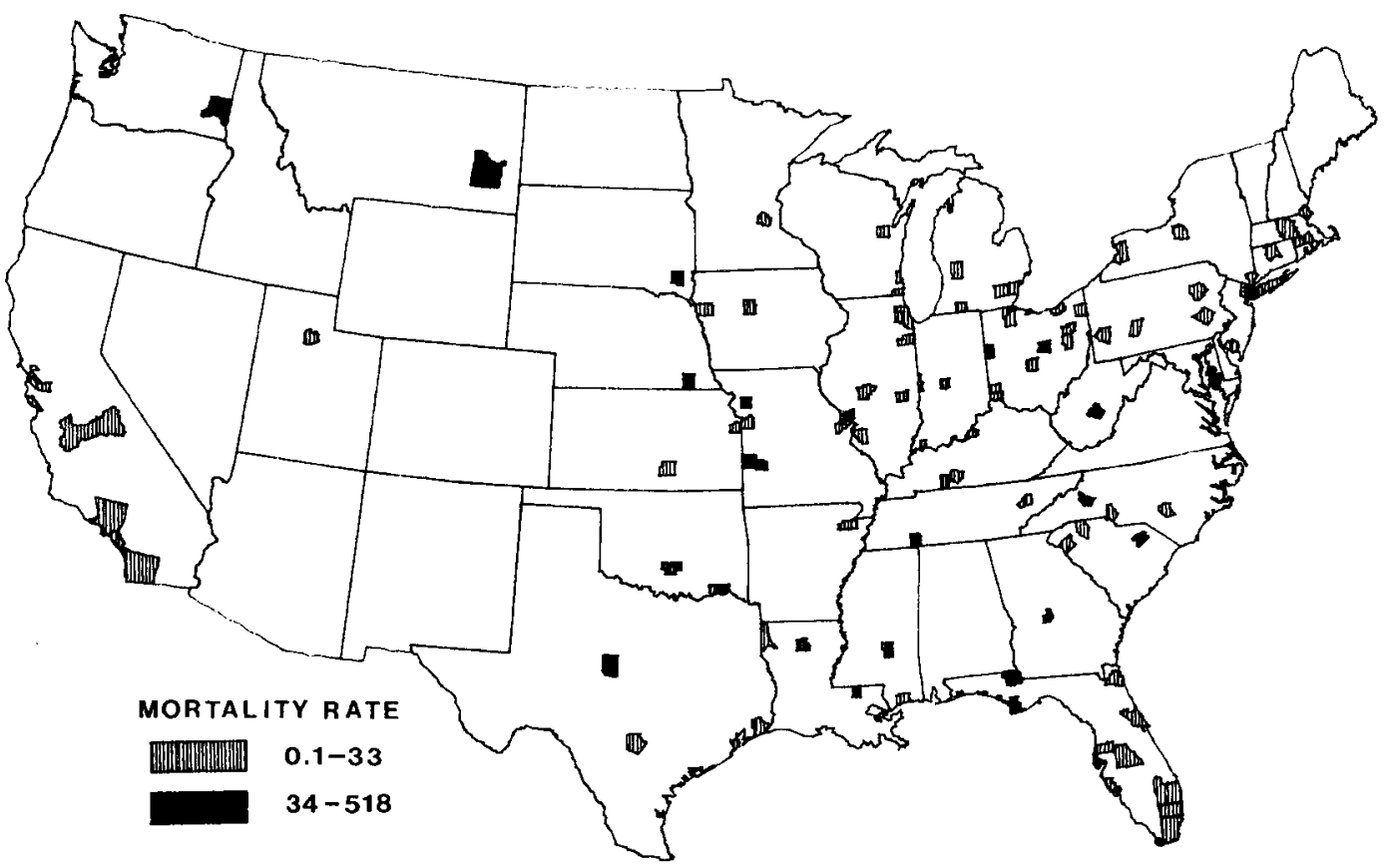

Fig 5. Deaths due to nasal cancer (ICDA 160) among white males, aged 65 years and over 1972 through 1974 . The mortality rates presented are the estimated average annual rates per 100,000 , age-adjusted to the 1970 United States population. The mortality rates of the white areas are zero or not estimable.

fig 5 the use of mortality rates helps but does not entirely eliminate the type of problem caused by the visual domination of counties with a concentration of population or a large land area.

\section{Next steps}

The five types of maps in this communication represent our first efforts to present both hazard and health effect information in a format that has three general uses. First the hazard and the mortality maps can be used independently to generate occupational health surveillance leads. Second the juxtaposition of potential hazard maps with maps that show causes of mortality may suggest new hypotheses that warrant in-depth study. Finally we believe computer-generated maps that utilize existing data files provide an efficient and effective means of disseminating information.

Methodologically much remains to be done. In addition to the problems associated with training and maintaining staff skilled in the use of automated cartographic techniques, we are also concerned with the following problems: (i) the need to increase the accuracy and completeness of both the hazard and the mortality data, (ii) the inability to use all of the information presently collected on death certificates, eg, multiple cause of death coding and information on the industry and occupation of the deceased, (iii) the lack of comparably coded county-level health parameters other than causes of death, eg, disease and disability data, and (iv) the need to develop computergenerated maps in a way that will encourage their use in priority setting, ie, in risk identification and risk estimation. We are in the process of moving ahead with the mapping surveillance program described in this paper.

\section{Acknowledgment}

The authors gratefully acknowledge the extensive cartographic mapping work that 
Mr W Yosafat and Mr E Dacey provided during the preparation of this paper.

\section{References}

1. Feinleib M, Fabsitz R. National Heart, Lung, Blood Instuite mapping project. In: US Department of Health, Education and Welfare. Proceedings of the 1976 workshop on automated cartography and epidemiology. Hyattsville, MD 1979. (US DHEW publication no (PHS) 79-1254).

2. Kleinman JC, Feldman JJ, Mugge RH. Geographic variation in infant mortality. Public health rep 91 (1976): 5, 423-432.

3. Mason TJ, McKay FW, Hoover R, Blot WJ, Fraumeni JF. Atlas of cancer mortality for US counties 1950-1969. US Department of Health Education and Welfare, Washington,
DC 1975. (DHEW publication no (NIH) 75780).

4. McKay FW. Automated cartography for cancer research. In: US Department of Health, Education and Welfare. Proceedings of the 1976 workshop on automated cartography and epidemiology. Hyattsville, MD 1979. (US DHEW publication no (PHS) 791254).

5. National Institute for Occupational Safety and Health. National occupational hazard survey. Volume I (Survey manual). Cincinnati, OH 1974. (DHEW (NIOSH) publication no $74-127$ ).

6. National Institute for Occupational Safety and Health. Formaldehyde: Evidence of carcinogenicity - Dec 23, 1980. Cincinnati, $\mathrm{OH}$ 1981. (US DHHS (NIOSH) publication no $81-111$ )

7. Rantanen J. Risk assessment and the setting of priorities in occupational health and safety. Scand $\mathrm{j}$ work environ health 7 (1981): suppl 4, 84-90. 